\title{
Constrained mixture modeling affects material parameter identification from planar biaxial tests
}

\author{
Lauranne Maes ${ }^{\mathrm{a}, *}$, Heleen Fehervary ${ }^{\mathrm{a}}$, Julie Vastmans ${ }^{\mathrm{a}}$, S. Jamaleddin \\ Mousavi $^{\mathrm{b}}$, Stéphane Avril ${ }^{\mathrm{b}}$, Nele Famaey ${ }^{\mathrm{a}}$ \\ ${ }^{a}$ Biomechanics Section, Mechanical Engineering Department, KU Leuven, Leuven, Belgium \\ ${ }^{b}$ Mines Saint-Etienne, Univ Lyon, Univ Jean Monnet, INSERM, U 1059 Sainbiose, Centre \\ CIS, F - 42023 Saint-Etienne, France
}

\begin{abstract}
The constrained mixture theory is an elegant way to incorporate the phenomenon of residual stresses in patient-specific finite element models of arteries. This theory assumes an in vivo reference geometry, obtained from medical imaging, and constituent-specific deposition stretches in the assumed reference state. It allows to model residual stresses and prestretches in arteries without the need for a stress-free reference configuration, most often unknown in patient-specific modeling.

A finite element (FE) model requires material parameters, which are classically obtained by fitting the constitutive model to experimental data. The characterization of arterial tissue is often based on planar biaxial test data, to which nonlinear elastic fiber-reinforced material parameters are fitted. However, the introduction of the constrained mixture theory requires an adapted approach to parameter fitting. Therefore, we introduce an iterative fitting method, alternating between nonlinear least squares parameter optimization and an FE prestressing algorithm to obtain the correct constrained mixture material state during the mechanical test.

We verify the method based on numerically constructed planar biaxial test data sets, containing ground truth sets of material parameters. The results show
\end{abstract}

${ }^{*}$ Corresponding author: lauranne.maes@kuleuven.be, KU Leuven, Biomechanics Section, Celestijnenlaan 300 - box 2419, 3001 Leuven (Belgium)

Preprint submitted to Journal of the Mechanical Behavior of Biomedical MaterialsApril 9, 2019 
that the method converges to the correct parameter sets in just a few iterations. Next, the iterative fitting approach is applied to planar biaxial test data of ovine pulmonary artery tissue. The obtained results demonstrate a convergence towards constrained mixture compatible parameters, which differ significantly from classically obtained parameters.

We show that this new modeling approach yields in vivo wall stresses similar to when using classically obtained parameters. However, due to the numerous advantages of constrained mixture modeling, our fitting method is relevant to obtain compatible material parameters, that may not be confused with parameters obtained in a classical way.

Key words: constrained mixture, deposition stretches, arterial tissue, constitutive modeling, parameter estimation

\section{Introduction}

\subsection{Presence of residual stresses}

In its in vivo state, an arterial segment is subjected to two kinds of external loading. The inner blood pressure, fluctuating between diastole and systole, 5 causes a load in the radial direction. Secondly, axial stretching during growth causes axial loading. Therefore, a shortening of the segment can be observed during excision, revealing the presence of axial prestretch [1. This excised, cylindrical configuration is freed from both types of external loads and is further referred to as the load-free configuration.

10 Bergel [2] discovered an uncoiling of the cylindrical configuration of an arterial segment after a radial cut. This suggested that residual circumferential stresses reside inside the load-free configuration [3, 4].

Chuong and Fung [5] found that the residual stresses homogenize the circumferential stress across the wall in its physiological condition. Rachev and

15 Greenwald [6] hypothesized that residual stresses lead to an optimal load bearing performance, enabling to comply with blood pressure alterations. This shows 
the importance of understanding and incorporating the phenomenon of residual stresses in computational models.

\subsection{Measuring residual stresses}

The radially cut configuration is most often considered to be stress-free [7]. After making a radial cut, an opening angle can be observed. In many cases, this angle is used as a measure for circumferential residual stresses as done by, e.g. Vaishnav and Vossoughi [8], Liu and Fung [7, Hong et al. [9] and Matsumoto et al. [10. However, Vossoughi et al. [11] gave evidence that a single radial cut cannot relieve the material from all stresses. Their studies show that the different layers in the arterial wall have different opening angles, such that more cuts are needed to further release the stresses. Therefore, the assumption of a radially cut stress-free state is inaccurate. Moreover, obtaining the opening angle as a measure for residual stresses is tedious work, requiring the excision of a segment of the artery of interest.

\subsection{Classical modeling of residual stresses}

Classical modeling frameworks and numerical simulations with arterial tissue require the knowledge of a stress-free reference state, to which all other configurations can be related with a deformation gradient. Multiple research groups

35 have used the concept of the opening angle to define this stress-free reference state. Analytical solutions that relate the intact load-free state to the opened stress-free state have been derived by Chuong and Fung [4] by assuming plane strain deformation. Taber and Eggers [12] considered a deformation where the wall thickness is constant and the neutral line is located at the middle. Holzapfel et al. [13] and Rachev and Greenwald [6] considered a hyperelastic, incompressible material, deforming by pure bending. An extension of this opening angle method for multiple layers was developed by Taber and Humphrey [14. Using the same assumption of a stress-free reference state, a number of numerical simulations were carried out, starting from the opened geometry. The load-free, 
intact geometry is then obtained by nodal displacement, as e.g. done by Gasser et al. [15], Raghavan et al. [16], Balzani et al. [17] and Famaey et al. [18].

There are a number of problems associated with this approach in the case of patient-specific modeling. The opening angle of an artery cannot be obtained nondestructively. Moreover, only cylindrical geometries can be consid-

so ered, whereas true arteries may deviate strongly from this regular shape, especially when one is interested in diseased vessels or aneurysms.

\subsection{Inverse methods}

As a solution, a number of groups have developed prestressing algorithms, designed to obtain a realistic stress-state. These algorithms are based on a known physiologically relevant internal pressure and pressurized geometry, that can be obtained noninvasively through medical imaging. De Putter et al. [19] and Weisbecker et al. [20] computed the prestressing deformation gradient, i.e. the deformation gradient relating the known in vivo geometry to the load-free geometry, with a backwards incremental method. At each iteration, the required pressure incrementation is mapped to the deformation needed to relate the known in vivo geometry to the current estimate of the load-free geometry, leading to an equilibrium between the in vivo geometry and pressure after convergence in the last increment.

An alternative approach was developed by Bols et al. [21], who proposed a backwards displacement method. At each iteration, the in vivo pressure is applied to the approximated load-free configuration. Subsequently, the resulting nodal displacement is subtracted from the known in vivo geometry. After convergence, a solution for a stress-free state is obtained, which can be pressurized to determine any physiological configuration of the artery.

Other prestressing algorithms were described by Raghavan et al. 22] and Gee et al. 23, 24. These algorithms allow the integration of prestress at diastole in finite element (FE) models, greatly enhancing the reliability of simulations of patient-specific cases. However, all previously mentioned algorithms are unable to reliably represent circumferential residual stresses in arterial tissue, because 
the application to cylindrical geometries yields a stress-free zero-pressure configuration [19, 20].

\subsection{Constrained mixture modeling}

The aforementioned algorithms assume that, while deforming together in the arterial wall, the different material constituents experience the same stretch levels. However, residual stresses likely originate from the fact that the constituents are deposited at different time points and experience different levels of stretch during multiple events of growth and remodeling [25, 26, 27]. Therefore, a new theoretical framework was proposed by Bellini et al. [28], based on the constrained mixture theory, first described by Humphrey and Rajagopal [29]. This framework accounts for the natural stress state of the individual constituents with constituent-specific deposition stretches, relating an in vivo reference configuration to the individual rest lengths of the constituents. Results show a reliable prediction of the zero-pressure configuration and the residual stresses, without the need of an opening angle or a stress-free reference configuration.

Moreover, the constrained mixture theory is increasingly used in the modeling of growth and remodeling in soft tissues, as done by [30, 31, 32, 33, 34, 35, 36, 37. In vivo, these remodeling processes occur through mass turnover of the different constituents 29]. Due to the microstructurally motivated nature of the constrained mixture theory, it offers a relevant framework for growth and remodeling algorithms.

The above modeling approach implies knowledge of the material parameters. This can be obtained, e.g. from planar biaxial tests, or from extension-inflation tests. However, the classical approaches to the parameter fitting of planar biaxial test data does not take the concept of residual stresses into account. They assume a stress-free configuration at the start of the test and a homogeneous stretch state of the material. On the other hand, the constrained mixture approach assumes a relevant in vivo reference state. We hypothesize that this assumption has an important influence on the resulting material parameters and propose an original solution to incorporate the constrained mixture modeling 

process.

Therefore, we introduce an iterative parameter fitting approach, alternating between a nonlinear least squares optimization and a prestressing FE simulation. This new approach allows to determine material parameters compatible with the constrained mixture theory, enabling a more biologically relevant approach for the modeling of healthy, matured arteries. In the present work, this framework is verified against numerically constructed planar biaxial data sets and tested on real experimental data, as explained in the following sections.

\section{Material and Methods}

The description of the method starts with a depiction of the considered material model. Subsequently, we explain how deposition stretches for the constrained mixture model are obtained and how they are integrated in the parameter fitting on planar biaxial test data. Finally, an explanation of the verification and testing approach is given.

\subsection{Material description}

An anisotropic, hyperelastic description of the artery's mechanical behavior can be made through an additive decomposition of a strain energy density function $\Psi$, with a contribution of the isotropic extracellular matrix material, mainly elastin, and the embedded collagen fibers, which are assumed to run along two preferred directions. This leads to the classic Gasser-Ogden-Holzapfel (GOH) formulation 38

$\Psi=C_{10}\left(I_{1, e}-3\right)+\frac{k_{1}}{2 k_{2}} \sum_{i=4,6}\left\{\exp \left\{k_{2}\left[\left(\kappa I_{1, c}+(1-3 \kappa) I_{i, c}\right)-1\right]^{2}\right\}-1\right\}, \quad i=4,6$,

with $C_{10}, k_{1}, k_{2}$ and $\kappa$ material constants. $I_{1, e}$ and $I_{1, c}$ are the first invariants of the right Cauchy-Green stretch tensors $\boldsymbol{C}_{e}$ and $\boldsymbol{C}_{c}$, applied to the elastin and the collagen constituents, respectively. $I_{i, c}$ is a pseudo-invariant of $\boldsymbol{C}_{c}$, and 
represents the stretch along each preferred fiber direction as

$$
I_{i, c}=M_{i} \cdot\left(\boldsymbol{C}_{c} \boldsymbol{M}_{i}\right), \quad i=4,6,
$$

with $\boldsymbol{M}_{i}=\left[\begin{array}{lll}0 & \cos \alpha_{i} & \sin \alpha_{i}\end{array}\right]$ the undeformed fiber orientation vector, where the order of the axes is given as $r$ (radial), $\theta$ (circumferential) and $z$ (axial). $\alpha_{i}$ is the mean angle of the fibers with respect to the circumferential direction of the artery in the $\theta, z$-plane.

Note that in a classical modeling approach,

$$
\boldsymbol{C}_{e}=\boldsymbol{C}_{c}=\boldsymbol{C}=\boldsymbol{F}^{T} \boldsymbol{F},
$$

where $\boldsymbol{F}$ is the total deformation gradient with respect to a stress-free reference geometry. Contrarily, in the constrained mixture theory,

$$
\boldsymbol{C}_{j}=\boldsymbol{F}_{j}^{T} \boldsymbol{F}_{j} \quad j=e, c,
$$

where

$$
\boldsymbol{F}_{j}=\boldsymbol{F G}_{j} \quad j=e, c
$$
is assumed to be zero for both fiber families in the media. The fiber dispersion, represented by $\kappa$ is assumed to be the same in both layers of the arterial wall. In total, there are five independent parameters, the medial $C_{10, M}$, the fiber 
parameters $k_{1}, k_{2}$ and $\kappa$ and the adventitial fiber angle $\alpha_{A}$ with respect to the circumferential direction. The used parameters are summarized in table 1 .

\begin{tabular}{ll}
\hline Media $(\mathrm{M})$ & Adventitia (A) \\
\hline$C_{10, M}$ & $C_{10, A}=0.1 \cdot C_{10, M}$ \\
$k_{1, M}=k_{1}$ & $k_{1, A}=k_{1}$ \\
$k_{2, M}=k_{2}$ & $k_{2, A}=k_{2}$ \\
$\kappa_{M}=\kappa$ & $\kappa_{A}=\kappa$ \\
$\alpha_{M}=0$ & $\alpha_{A}$ \\
\hline
\end{tabular}

Table 1: Adventitial and medial material parameters.

\subsection{An algorithm to find deposition stretches}

According to Bellini et al. [28, the constituents in the diastolic reference configuration of the artery experience different prestretches. These stretches are introduced through deposition stretch tensors $\boldsymbol{G}_{e}$ and $\boldsymbol{G}_{c}$ for elastin and collagen respectively. These deformation gradients are defined in accordance with [30] and as described below. Collagen fibers may have a fast turnover rate and are deposited at a preferred stretch state [29]. Therefore, we assume a constant and known deposition stretch $g_{c}$ for collagen along the fiber direction [28, 30, 40, justified by the fact that we focus on healthy matured arteries. An approximate value of collagen prestretch of 1.1 was found by Bellini et al. 28, and used in the methods explained further on. For a certain fiber family with orientation vector $\boldsymbol{M}, \boldsymbol{G}_{c}$ may then be written as 32

$$
\boldsymbol{G}_{c}=g_{c} \boldsymbol{M} \otimes \boldsymbol{M}+\frac{1}{\sqrt{g_{c}}}(\boldsymbol{I}-\boldsymbol{M} \otimes \boldsymbol{M}),
$$

where $\boldsymbol{I}$ is the identity tensor.

On the other hand, elastin is deposited at an early stage of development and is very stable. As a consequence, it is stretched during growth. $\boldsymbol{G}_{e}$ therefore varies from material point to material point and balances out the reference diastolic configuration and corresponding pressure. Considering a known and constant axial deposition stretch $g_{e, a x}$ and an isochoric deformation, the solution is unique. 
The unknown deposition stretch values are determined iteratively, similarly to [30] and [40]. A finite element (FE) method, schematically represented in figure 1. is implemented by initially considering a load- and stress-free diastolic configuration. In a first step, the diastolic pressure is applied to the geometry, while $\boldsymbol{G}_{c}$ and an initial elastin deposition stretch tensor

$$
\boldsymbol{G}_{e}=\left[\begin{array}{ccc}
\frac{1}{\sqrt{g_{e, a x}}} & 0 & 0 \\
0 & \frac{1}{\sqrt{g_{e, a x}}} & 0 \\
0 & 0 & g_{e, a x}
\end{array}\right]
$$

are applied to the material, where $g_{e, a x}$ represents the axial deposition stretch

150 of elastin.

In all following simulation steps, the resulting deformation gradient with respect to the diastolic reference state in each material point is multiplied to the existing $\boldsymbol{G}_{e}$, from which the shear terms are removed, to cause an apparent stiffening and recoiling towards the reference configuration. This is repeated until the current configuration matches the initial configuration, i.e. when the average absolute nodal displacement is below a threshold defined as $1 \%$ of the diastolic wall thickness [40]. In practice, the algorithm is run using a user material subroutine (UMAT) in Abaqus/Standard 2017 (Dassault Systèmes Simulia Corp., Providence Rhode Island, USA). See the appendix for further details on the implementation. This algorithm is further referred to as the "prestressing algorithm'. 


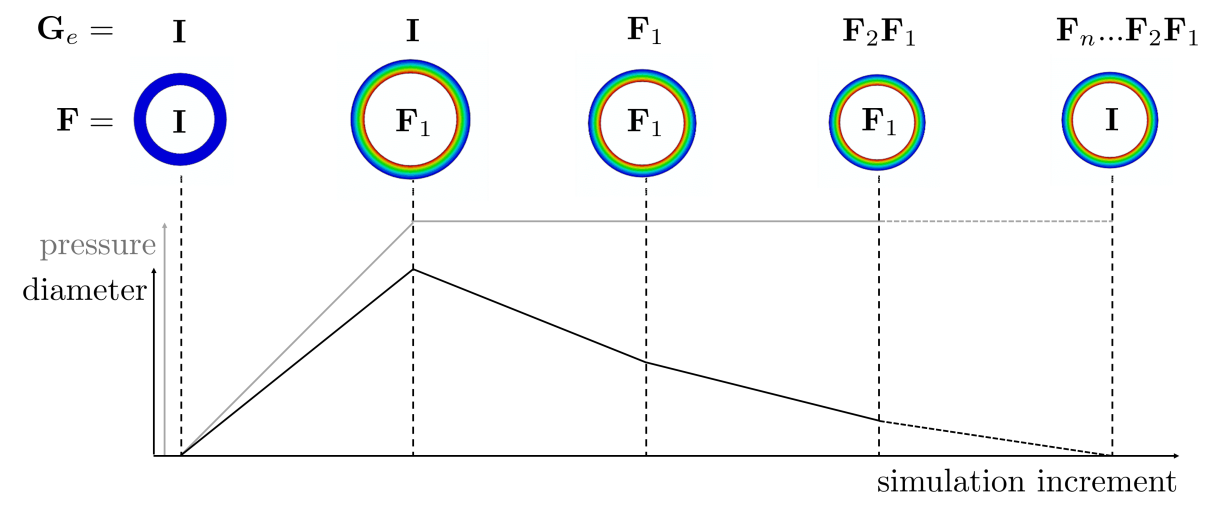

Figure 1: Schematic representation of the prestressing algorithm to obtain the elastin deposition stretches. $\boldsymbol{G}_{e}$ is the deformation gradient accounting for the elastin deposition stretch and $\boldsymbol{F}$ is the deformation of the artery with respect to its diastolic reference configuration. Adapted from 30.

\subsection{Parameter fitting of planar biaxial tests}

\subsubsection{Classical approach}

During a planar biaxial test, a flat squared sample of material is attached at its four sides to the actuators of the testing machine. This way, the displacement in two perpendicular directions can be applied independently. The normal forces applied by the actuators can be measured directly and are further referred to as the experimental forces, $f_{i i}^{e x p}$, with $i$ indicating one of the two test directions. The deformation of the sample is usually obtained through image processing, where four markers or a speckle pattern in the central region of the sample are tracked, from which the in-plane deformation gradient can be derived.

The obtained deformation gradient $\boldsymbol{F}$ at each sampling time point of the experiment is used to estimate the required first Piola-Kirchhoff stress as

$$
\boldsymbol{P}^{\text {mod }}=J \boldsymbol{\sigma}^{\text {mod }} \boldsymbol{F}^{T}
$$

with $\boldsymbol{\sigma}^{\text {mod }}$ the Cauchy stress calculated as

$$
\boldsymbol{\sigma}^{m o d}=\frac{1}{J} \frac{\partial \Psi}{\partial \boldsymbol{F}} \boldsymbol{F}^{T}-p \boldsymbol{I}
$$


Here, $\boldsymbol{F}$ pertains to the total deformation during the biaxial test and $J=$ $\operatorname{det}(\boldsymbol{F}) . \Psi$ is the strain energy density function of the material as described in equation 1 , with a currently unknown set of material parameters $\boldsymbol{p}=\left[C_{10, M}, k_{1}, k_{2}, \kappa, \alpha_{A}\right]$. The hydrostatic pressure $p$ multiplied with the identity tensor $\boldsymbol{I}$ arises from a constraint of incompressibility and is calculated by assuming a zero out-of-plane stress. The first Piola-Kirchhoff stress is then transformed to the model force by multiplication with a reference surface as

$$
f_{i j}^{m o d}=P_{i j} A_{j} \quad i, j=1,3,
$$

where $f_{i j}$ is the force along the $i$-th direction, acting on the $j$-th surface, and $A_{j}$ the undeformed surface upon which the force is acting.

To obtain the unknown material parameters, the following objective function is minimized:

$$
\min _{\boldsymbol{p}} \sum_{j=1}^{n}\left[\left(f_{11}^{\bmod }\left(t_{j}\right)-f_{11}^{e x p}\left(t_{j}\right)\right)^{2}+\left(f_{22}^{\bmod }\left(t_{j}\right)-f_{22}^{e x p}\left(t_{j}\right)\right)^{2}\right],
$$

with $t_{j}$ the sampling time points of the experiment and $f_{i i}^{e x p}\left(t_{j}\right)$ the experimentally measured force. In the experiments that follow, we used a nonlinear least squares optimization routine lsqnonlin in Matlab 2017a (The Mathworks Inc., Natick, Massachusetts, USA), along with the multistart function with 10 initial parameter sets in order to obtain the global minimun.

In the classical biaxial fitting approach, no deposition stretches are modeled, and all constituents of the mixture are assumed to feel the same deformation gradient $\boldsymbol{F}=\boldsymbol{F}_{\text {biax }}$. As such, the configuration at the beginning of the biaxial test is assumed to be the stress-free reference configuration.

\subsubsection{Constrained mixture-compatible fitting}

Considering diastole to be the reference configuration, a testing sample prepared for a biaxial test has undergone depressurization, release of axial prestretch $\boldsymbol{F}_{\text {release }}$, opening to release circumferential stresses and further flattening to a square patch $\boldsymbol{F}_{\text {flatten }}$. Considering also the deposition stretches of collagen and elastin that were present at diastole, it is highly unlikely that the 
constituents are stress-free at the start of the biaxial test. Rather, during the biaxial test, they will have the following deformation:

$$
\boldsymbol{F}_{j}=\boldsymbol{F}_{\text {biax }} \boldsymbol{F}_{\text {flatten }} \boldsymbol{F}_{\text {release }} \boldsymbol{G}_{j}, \quad j=e, c .
$$

Hence, when calculating the Cauchy stresses to obtain the model forces in the objective function of equation 11, this total deformation for each of the constituents should be taken into account, i.e.

$$
\boldsymbol{\sigma}^{\text {mod }}=\frac{1}{J} \frac{\partial \Psi}{\partial \boldsymbol{F}} \boldsymbol{F}^{T}-p \boldsymbol{I}=\frac{1}{J}\left(\frac{\partial \Psi}{\partial \boldsymbol{F}_{e}}: \frac{\partial \boldsymbol{F}_{e}}{\partial \boldsymbol{F}}+\frac{\partial \Psi}{\partial \boldsymbol{F}_{c}}: \frac{\partial \boldsymbol{F}_{c}}{\partial \boldsymbol{F}}\right) \boldsymbol{F}^{T}-p \boldsymbol{I} .
$$

Note however, that the deposition stretch tensors as well as the deformations due to depressurization, release of axial prestretch, opening and flattening depend on the geometry and material properties of the tested sample, the latter being exactly what we are trying to optimize. Hence, an iterative scheme is proposed to obtain these constrained mixture-compatible (CMC) material parameters, as shown in figure 2 and described below.

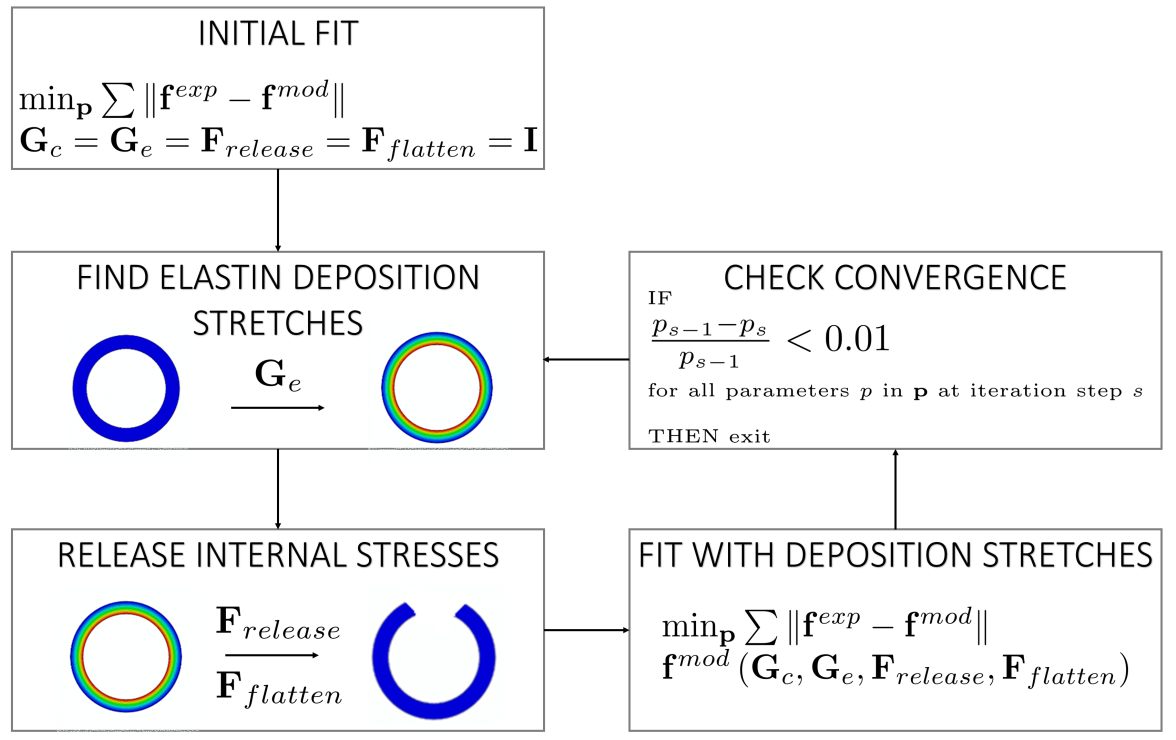

Figure 2: Schematic representation of the iterative parameter fitting approach, compatible with the constrained mixture theory. 
In the initialization step, a classical parameter fitting scheme as described in section 2.3.1 is applied, yielding a set of material parameters. These parameters are then used in the prestressing algorithm, described in section 2.2 , to obtain accompanying deposition stretches. At the end of the algorithm, the resulting pressurized artery is depressurized and axial stresses are released. The resulting deformation gradient is stored as $\boldsymbol{F}_{\text {release }}$. Next, a radial cut is made through the mesh to release the residual circumferential stresses. The resulting deformation in this last step is assigned to $\boldsymbol{F}_{\text {flatten }}$, assuming that the circumferential stretches from this nearly stress-free configuration to a flat unloaded configuration are negligible.

Now that estimates of $\boldsymbol{F}_{\text {flatten }}, \boldsymbol{F}_{\text {release }}, \boldsymbol{G}_{e}$ and $\boldsymbol{G}_{c}$ are available, a new parameter fitting procedure is performed, where this time the model stresses are calculated as in equation 13. It is important to note that $\boldsymbol{F}_{\text {flatten }}, \boldsymbol{F}_{\text {release }}$ and $\boldsymbol{G}_{e}$ are not constant through the thickness of the artery. Therefore, the stress field of the biaxial sample is not homogeneous through the thickness and multiple material layers must be considered during the parameter fitting. One average deposition stretch tensor $\boldsymbol{G}_{e}$ per layer of finite elements is extracted from the simulation. The combined deformation gradient $\boldsymbol{F}_{\text {flatten }} \boldsymbol{F}_{\text {release }}$ is determined at the end of the mentioned simulations with respect to the initial diastolic configuration. The axial shortening is extracted and an average circumferential stretch per layer is determined from the total change in length of these element edges. This results in an overall axial stretch $\lambda_{z}$ and one circumferential stretch $\lambda_{\theta}$ per element layer. Considering an isochoric deformation, $\boldsymbol{F}_{\text {flatten }} \boldsymbol{F}_{\text {release }}$ is then written as

$$
\boldsymbol{F}_{\text {flatten }} \boldsymbol{F}_{\text {release }}=\left[\begin{array}{ccc}
\frac{1}{\lambda_{\theta} \lambda_{z}} & 0 & 0 \\
0 & \lambda_{\theta} & 0 \\
0 & 0 & \lambda_{z}
\end{array}\right] .
$$

The model force is calculated at each layer as explained above and these are summed to obtain an overall model force to be matched to the experimentally measured forces. With the newly obtained material parameters, the prestressing 210 algorithm is rerun, followed by the calculation of $\boldsymbol{F}_{\text {release }}$ and $\boldsymbol{F}_{\text {flatten }}$. This 
loop is repeated until convergence of the material parameters, i.e. until the relative difference between subsequent parameters is smaller than $1 \%$ for each of the five independent fitted parameters.

\subsection{Verification study}

The constrained mixture-compatible (CMC) parameter fitting approach described above is verified against numerically constructed biaxial test data. This verification study is based on six sets of ground truth parameters, given in table 2. The five independent parameters are given. The elastin stiffness in the media $C_{10, M}$ is considered ten times higher than the elastin stiffness in the adventitia and the fibers are considered to run approximately in the circumferential direction in the media, whereas the fiber angle in the adventitia $\alpha_{A}$ can be different from zero.

\begin{tabular}{lccccccc}
\hline set & $C_{10, M}[\mathrm{MPa}]$ & $k_{1}[\mathrm{MPa}]$ & $k_{2}[-]$ & $\kappa[-]$ & $\alpha_{A}[\mathrm{rad}]$ & $g_{e, a x}[-]$ & $g_{c}[-]$ \\
\hline 1 & 0.5 & 0.1 & 10.0 & 0.2 & 0.7 & 1.1 & 1.05 \\
2 & 0.2 & 0.5 & 2.0 & 0.2 & 0.7 & 1.1 & 1.05 \\
3 & 0.5 & 0.1 & 10.0 & 0.0 & 1.2 & 1.1 & 1.05 \\
4 & 0.5 & 0.1 & 10.0 & 0.2 & 0.7 & 1.3 & 1.1 \\
5 & 0.2 & 0.05 & 10.0 & 0.2 & 0.7 & 1.3 & 1.1 \\
6 & 0.2 & 0.05 & 10.0 & 0.0 & 1.2 & 1.1 & 1.05 \\
\hline
\end{tabular}

Table 2: The six sets of material parameters used in the verification study for the CMC parameter fitting, with corresponding axial elastin deposition stretch $g_{e, a x}$ and collagen deposition stretch $g_{c}$.

These parameter sets are used in FE simulations in Abaqus. The diastolic geometry of a human thoracic aorta is modeled as a cylinder with an inner radius of $15.0 \mathrm{~mm}$. The media and adventitia thicknesses are $1.18 \mathrm{~mm}$ and $0.93 \mathrm{~mm}$ respectively [39]. Due to symmetry of the problem, only half of the cylinder is modeled in Abaqus and symmetry boundary conditions are assigned at the appropriate symmetry plane. The mesh contains 12096 hexahedral, fully integrated, hybrid elements (C3D8H). The total of four medial and four adven- 
titial element layers ensures mesh convergence. The axial planes are fixed and a diastolic pressure of $10 \mathrm{kPa}$ is applied to the inner surface of the cylinder. All deposition stretches are determined as explained in section $2.2, \quad \boldsymbol{F}_{\text {release }}$ and $\boldsymbol{F}_{\text {flatten }}$ are obtained as in section 2.3.2.

A second simulation starts from a cuboid geometry, composed out of 512 C3D8H elements with a total of eight element layers, representing the arterial test sample at the beginning of a biaxial test. The thickness is set according to the resulting thickness after the simulation of the radial cut. The same material properties are assigned to this mesh, taking into account the difference between the media and adventitia. In addition, all obtained layer-specific deposition 240 stretches and deformation gradients are assigned to the material, i.e. $\boldsymbol{F}_{\text {release }}$, $\boldsymbol{F}_{\text {flatten }}$ and $\boldsymbol{G}_{e}$.

An ideal biaxial test is then simulated by assigning displacement increments in the circumferential and axial direction in three different ratios of maximal displacement causing up to approximately $25 \%$ stretch in the two test directions, 245 i.e. $1: 1,0.5: 1$ and 1:0.5. Subsequently, the resulting reaction forces at the displacement sites and the nodal coordinates of the marker nodes are extracted at each increment, resulting in numerically constructed biaxial test data sets. Figure 3 represents the workflow followed to obtain these data sets. Next, both parameter fitting approaches described in section 2.3 are applied to the obtained data sets. Classical material parameters are obtained in the first iteration of the fitting approach and CMC parameters are obtained after convergence. 


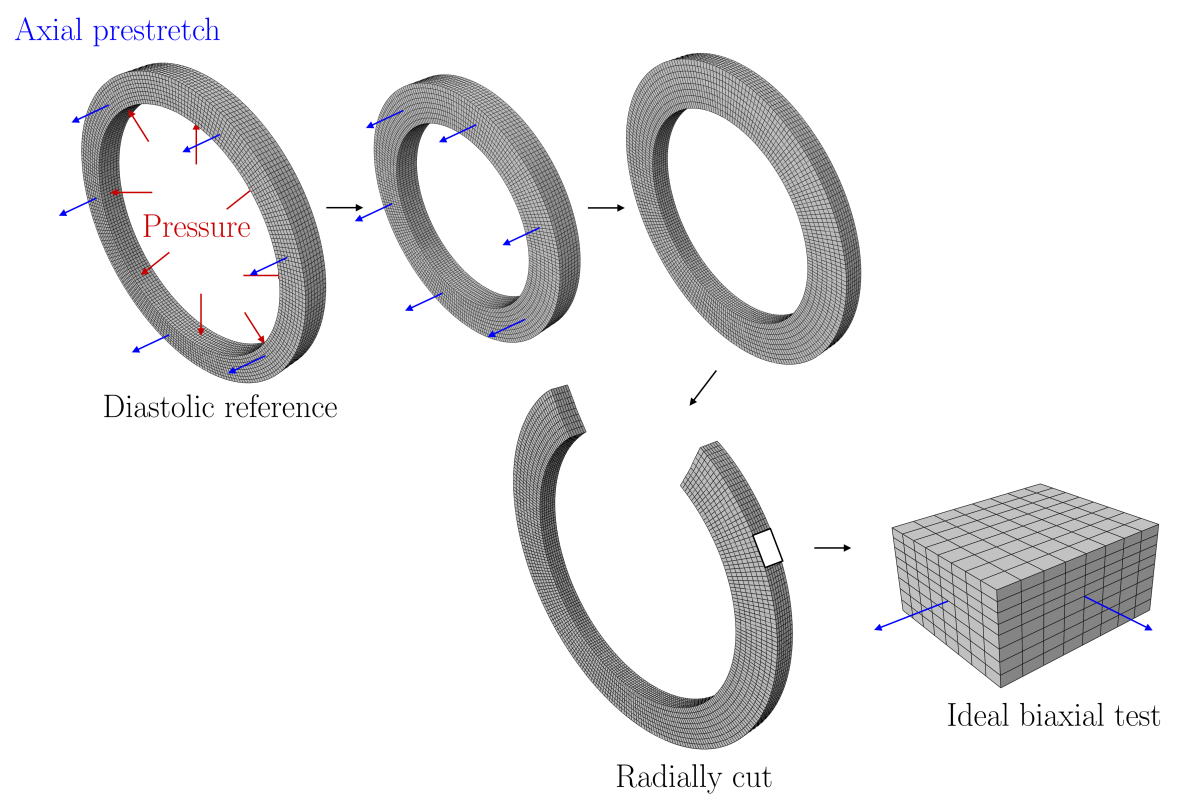

Figure 3: Schematic representation of the followed steps in the verification approach to obtain numerically constructed biaxial test data sets.

\subsection{Application to experimental data}

\subsubsection{Parameter fitting}

After verification, the CMC parameter fitting approach was applied to actual test data, obtained from Vastmans et al. [41. A tissue sample of a pulmonary artery was subjected to a planar biaxial tensile test, where the circumferential and axial direction were aligned according to the two test directions. The sample was loaded up to $1.2 \mathrm{~N}$ in three different load ratios between the circumferential load and the axial load, i.e. 1:1, 0.5:1 and 1:0.5. More information on the harvesting and preparation of this arterial tissue sample and about the applied testing protocol can be found in [41] (sheep 1983, pulmonary sample 2, P1). During the same biaxial test, four markers were manually attached to the tissue and tracked, from which $\boldsymbol{F}_{\text {biax }}$ is obtained at each loading point as explained in section 2.3 .1 . 
For the CMC parameter fitting approach, an FE model of a half cylinder is built, corresponding to the geometry of the considered sheep pulmonary artery. The diameter of the artery at diastole is set to $19.4 \mathrm{~mm}$, i.e. the diameter measured in vivo in [41. The ex situ sample thickness is reported to be $3.20 \mathrm{~mm}$ [41. Based on trial-and-error, the in vivo thickness of the artery is estimated at $2.50 \mathrm{~mm}$. The adventitia-to-media thickness ratio is roughly estimated at one, based on findings by [42], such that each layer thickness is set to $1.25 \mathrm{~mm}$ in the diastolic configuration. The length of the cylinder is set to $2.0 \mathrm{~mm}$.

The mesh is built out of $5136 \mathrm{C} 3 \mathrm{D} 8 \mathrm{H}$ elements. Both the media and the adventitia contain four element layers, such that eight material layers with different deposition stretches can be identified. The total of eight layers ensure mesh convergence. The same boundary conditions as in section 2.4 are applied to the model in order to obtain $\boldsymbol{G}_{e}, \boldsymbol{F}_{\text {release }}$ and $\boldsymbol{F}_{\text {flatten }}$ at each iteration of the fitting. The diastolic pulmonary blood pressure is estimated at $2 \mathrm{kPa}$ [30]. The axial elastin deposition stretch $g_{e, a x}$ is set to 1.16, i.e. an approximate 280 axial prestretch obtained from unpublished results related to [41]. The collagen deposition stretch $g_{c}$ is set to 1.1, a value found by [28] for mouse carotid arteries.

\subsubsection{Comparison of the classical and constrained mixture modeling approach}

A comparison of the wall stresses obtained with the constrained mixture modeling approach and the classical approach for the modeling of the sheep pulmonary artery can be made after material parameters for both are obtained. The classical material parameters are obtained in the first iteration of the developed fitting approach, whereas the CMC parameters are obtained after convergence of the entire procedure. In the case of the constrained mixture approach, this final parameter set is used in combination with the same FE model as in the previous section, to obtain the assumed diastolic reference state of the sheep artery according to section 2.2 In a next simulation step, the deposition stretches are kept constant and the pressure is increased to a pulmonary systolic pressure level of $3 \mathrm{kPa}$. 
The classical modeling approach considers the radially cut configuration as a stress-free reference state. Therefore, another FE model of the physiologically loaded artery is built, starting from this geometry, obtained by removing the appropriate boundary conditions after the final iteration of the CMC parameter fitting to simulate the excision and radial cut of the artery. This ex vivo geometry was approximated by a cylindrical section with a radius of $11.93 \mathrm{~mm}$ and an opening angle $\Theta$ of approximately $54^{\circ}$, as defined in figure 4 . The material is defined by the same UMAT in Abaqus, where $\boldsymbol{G}_{e}=\boldsymbol{G}_{c}=\boldsymbol{I}$, in combination with the material parameters obtained at the first iteration of the fitting procedure. The initial material orientations are determined by fitting a circle through the nodes of the new reference geometry in a plane perpendicular to the axial direction, following the approach by Pratt [43]. The fibers are assumed to run in a plane perpendicular to the radial direction obtained from the fitting of the circle. In a first simulation step, the opening angle is closed. Subsequently, an axial displacement of the top of the cylinder is imposed, while the bottom is fixed in the axial direction, in order to restore the axial prestretch. The level of displacement is determined based on the axial recoil that was observed earlier. In a next step, the diastolic blood pressure is applied to the inner surface, and is then increased to the systolic level. A schematic overview of the simulation steps for both approaches is shown in figure 4

From both simulation approaches, the Cauchy stresses in all directions at diastole and systole are extracted. The obtained values are compared and shown in section 3.2 .2 in order to assess the similarity. Finally, both FE models are discussed in section 4.3 . 
CONSTRAINED MIXTURE MODELING

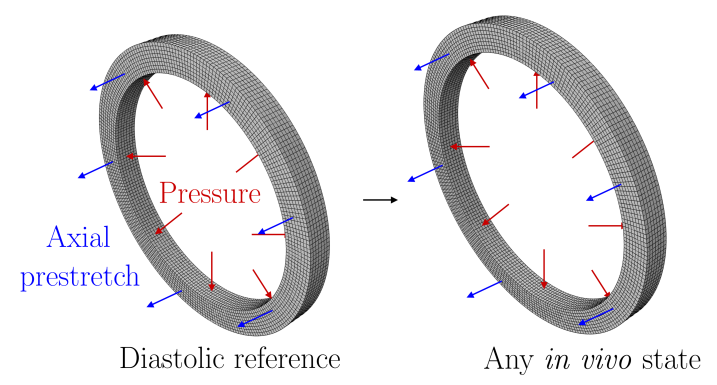

CLASSICAL MODELING

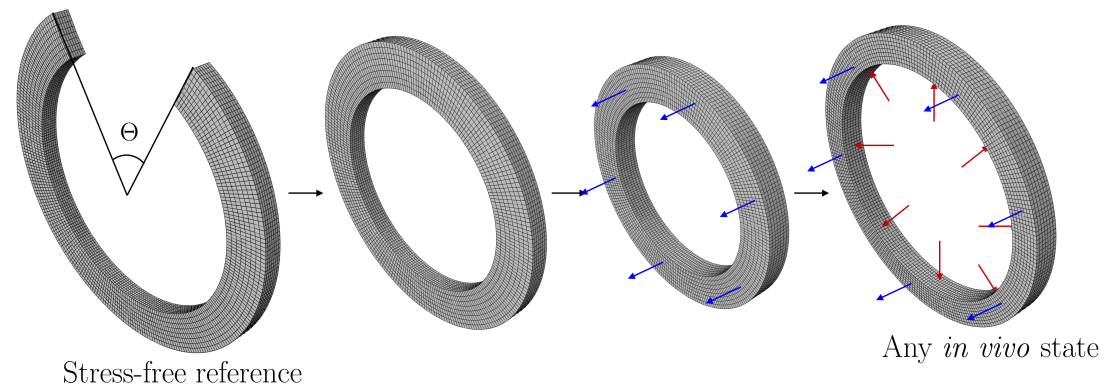

Figure 4: Schematic representation of the constrained mixture modeling approach and the classical modeling approach.

\section{Results}

\subsection{Verification study}

Table 3 gives an overview of the results of the verification study for the CMC parameter fitting method accounting for residual stresses. The fitted parameter sets are shown and compared to the true parameters from table 2 via the mean absolute percentage error (MAPE), computed as

$$
\frac{100 \%}{5} \sum_{i=1}^{5}\left|\frac{p_{i, \text { true }}-p_{i}}{p_{i, \text { true }}}\right|
$$

for all estimated parameters $p_{i}$ and corresponding ground truth parameters $p_{i, t r u e}$. The 'classical' parameters are obtained by considering a fully stress-free 
test sample at the beginning of the biaxial test. These parameters are also the results of the first iteration of the CMC fitting procedure. After convergence of this procedure, the CMC parameters are obtained, after a certain required number of iterations (nb. it.). Remember that the value of $C_{10, A}$ in the adventitia is dependent on the value in the media and ten times smaller. Therefore it is not shown in the table. The fiber angle in the media is always considered zero and hence not fitted nor shown.

\begin{tabular}{|c|c|c|c|c|c|c|c|c|}
\hline set & & nb. it. & $C_{10, M}[\mathrm{MPa}]$ & $k_{1}[\mathrm{MPa}]$ & $k_{2}[-]$ & $\kappa[-]$ & $\alpha_{A}[\mathrm{rad}]$ & MAPE [\%] \\
\hline \multirow[t]{3}{*}{1} & true & & 0.5000 & 0.1000 & 10.0000 & 0.2000 & 0.7000 & \\
\hline & classical & & 0.4999 & 0.0732 & 8.2860 & 0.1216 & 0.5949 & 19.6 \\
\hline & $\mathrm{CMC}$ & 4 & 0.5000 & 0.1001 & 9.9969 & 0.2000 & 0.6999 & 0.03 \\
\hline \multirow[t]{3}{*}{2} & true & & 0.2000 & 0.5000 & 2.0000 & 0.2000 & 0.7000 & \\
\hline & classical & & 0.1787 & 0.3323 & 1.7366 & 0.1394 & 0.7373 & 18.6 \\
\hline & $\mathrm{CMC}$ & 4 & 0.2001 & 0.5003 & 2.0031 & 0.2001 & 0.6997 & 0.07 \\
\hline \multirow[t]{3}{*}{3} & true & & 0.5000 & 0.1000 & 10.0000 & 0.0000 & 1.2000 & \\
\hline & classical & & 0.7375 & 0.3349 & 13.9997 & 0.0000 & 0.0000 & 84.5 \\
\hline & $\mathrm{CMC}$ & 9 & 0.4998 & 0.1001 & 9.9994 & 0.0000 & 1.2003 & 0.03 \\
\hline \multirow[t]{3}{*}{4} & true & & 0.5000 & 0.1000 & 10.0000 & 0.2000 & 0.7000 & \\
\hline & classical & & 0.5366 & 0.1601 & 10.0751 & 0.0590 & 0.0000 & 47.7 \\
\hline & $\mathrm{CMC}$ & 8 & 0.4997 & 0.1003 & 9.9910 & 0.1998 & 0.6997 & 0.12 \\
\hline \multirow[t]{3}{*}{5} & true & & 0.2000 & 0.0500 & 10.0000 & 0.2000 & 0.7000 & \\
\hline & classical & & 0.2092 & 0.0323 & 7.2787 & 0.0515 & 0.0000 & 48.3 \\
\hline & $\mathrm{CMC}$ & 9 & 0.2000 & 0.0500 & 9.9840 & 0.1998 & 0.6997 & 0.06 \\
\hline \multirow[t]{3}{*}{6} & true & & 0.2000 & 0.0500 & 10.0000 & 0.0000 & 1.2000 & \\
\hline & classical & & 0.2325 & 0.0110 & 17.6526 & 0.0000 & 0.9336 & 38.6 \\
\hline & $\mathrm{CMC}$ & 5 & 0.2000 & 0.0500 & 10.0012 & 0.0000 & 1.2000 & $\sim 0$ \\
\hline
\end{tabular}

Table 3: The results of the validation study: the true parameters, the fitted parameters with the classical approach and the CMC parameters obtained iteratively by taking into account residual stresses. The number of iterations is indicated (nb. it.) and the mean absolute average error (MAPE) of the fitted parameters, as compared to the true values. 
Figure 5 shows how the material parameters evolve through the iterations for parameter set number 6 . The full line represents the obtained parameters at each iteration step, which are compared to the dashed line, representing the 'true' parameters. The evolution of $\kappa$ is not shown since it was zero at each iteration for this parameter set. The figure reveals a clear convergence towards the 'true' parameters after 5 iterations.
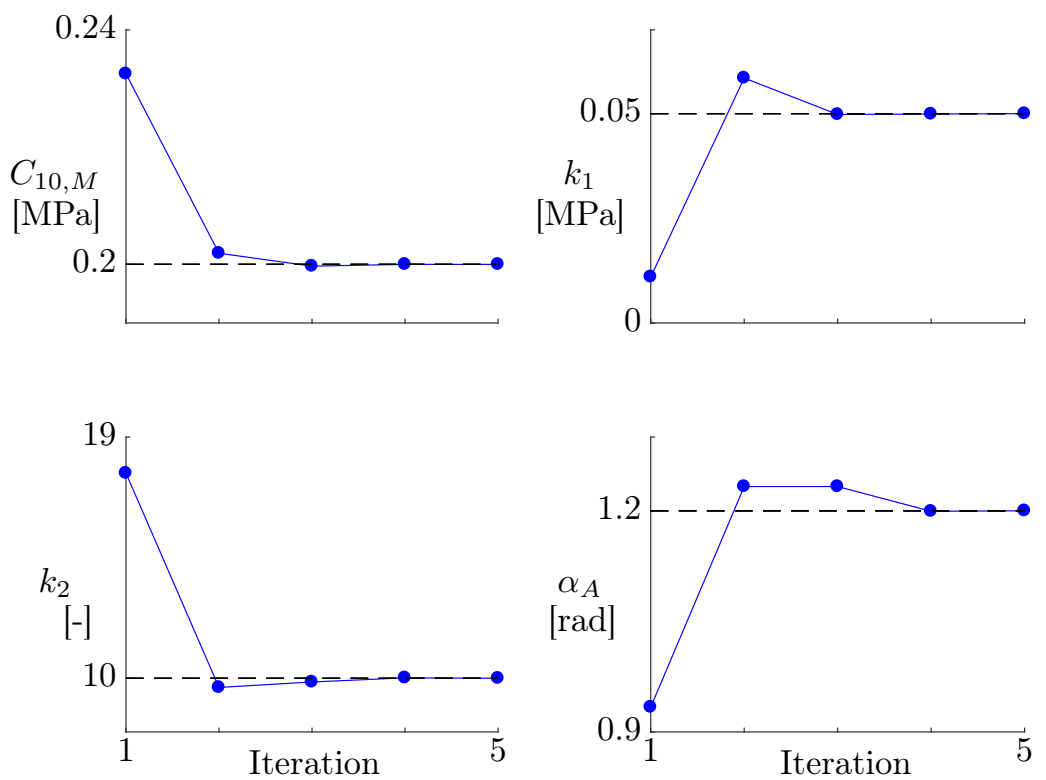

Figure 5: Evolution of the fitted material parameters during the iterative fitting approach (full line) of set number 6. Convergence towards the 'true' parameters (dashed line) can be observed after 5 iterations.

\subsection{Application to experimental data}

\subsubsection{Parameter fitting}

The iterative parameter fitting approach was tested on actual planar biaxial test data obtained on sheep pulmonary artery tissue. The results are given in table 4 . The medial stiffness parameter $C_{10, M}$ is given, as well as the fiber 
parameters $k_{1}$ and $k_{2}$, the fiber dispersion parameter $\kappa$ and the adventitial fiber orientation parameter $\alpha_{A}$, for two cases. First, these parameters are obtained after a classical fitting, based on the two-layered constitutive model, and second, after convergence of the iterative CMC fitting approach.

Figure 6 shows the evolution of the material parameters in function of the iteration number. Iteration number one corresponds to the initial fitting, according to the classical approach of obtaining material parameters. The fit of the model data to the experimental data at the first and last iteration is shown in figure 7. The initial fit is slightly better with a normalized root-mean-square error (NRMSE), a measure for the objective function of the optimization, of 0.1165 versus 0.1177 at the final fit.

\begin{tabular}{lccccccc}
\hline & nb. it. & $C_{10, M}[\mathrm{MPa}]$ & $k_{1}[\mathrm{MPa}]$ & $k_{2}[-]$ & $\kappa[-]$ & $\alpha_{A}[\mathrm{rad}]$ & NRMSE [-] \\
\hline classical & & 0.0115 & 0.0027 & 11.81 & 0.0949 & 0.7112 & 0.1165 \\
CMC & 7 & 0.0099 & 0.0126 & 21.31 & 0.2034 & 0.6329 & 0.1177 \\
\hline
\end{tabular}

Table 4: The results of the fitting to the actual planar biaxial data from sheep pulmonary artery tissue. The classical set of parameters is obtained by assuming a stress-free reference state at the beginning of the test. The CMC set of parameters is obtained by taking into account residual stresses and an in vivo reference state. The required number of iterations is given, as well as the obtained parameters and the NRMSE for both fits. 

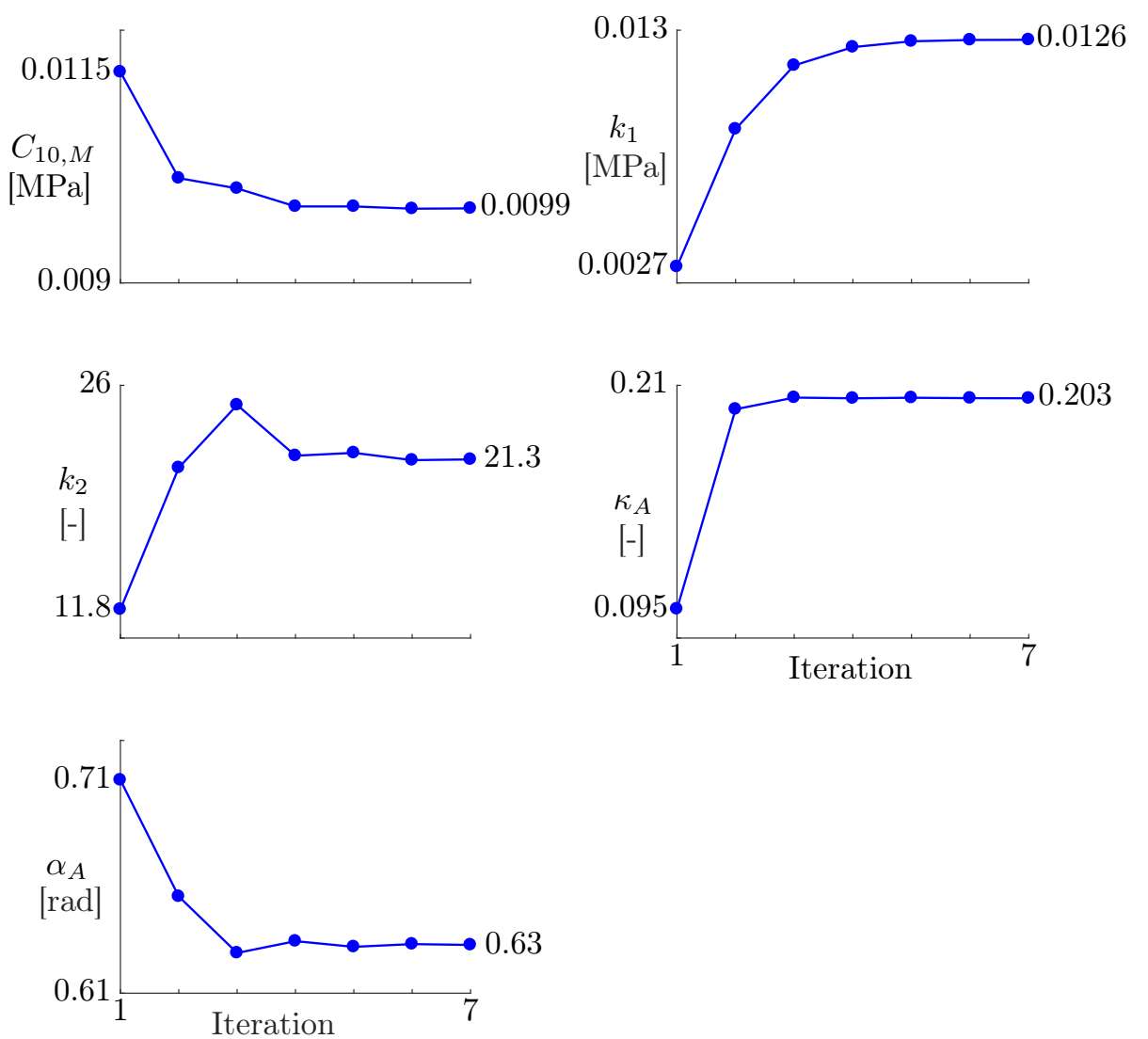

Figure 6: Evolution of the fitted material parameters during the iterative CMC approach applied to planar biaxial test data from sheep pulmonary artery tissue. 

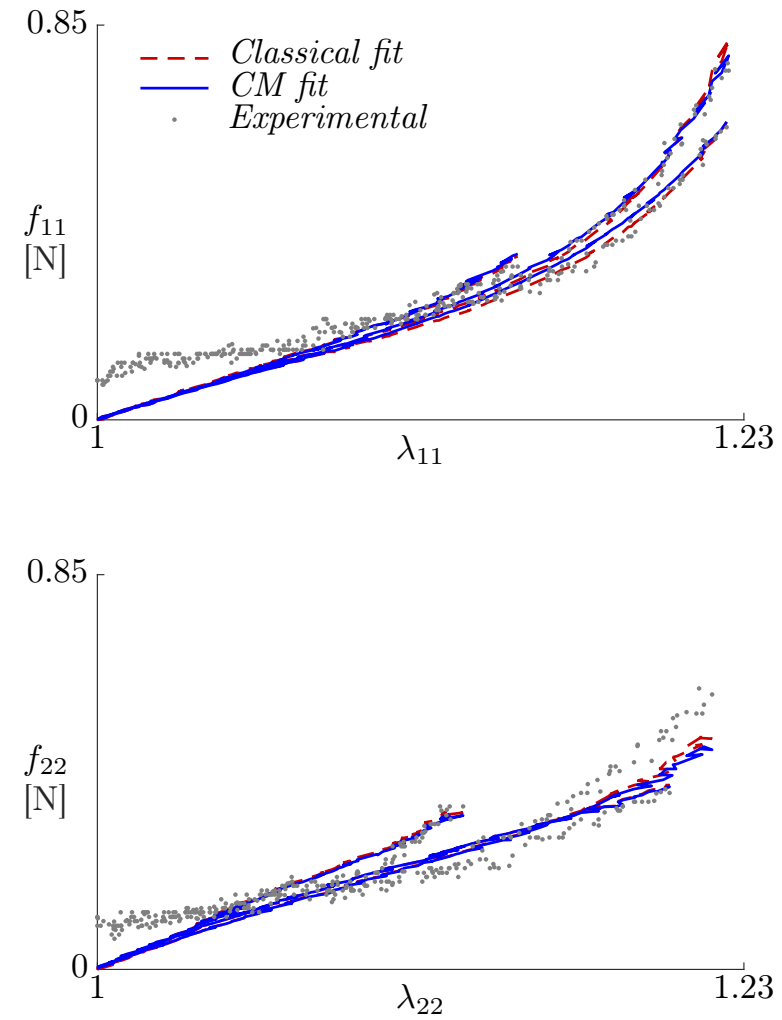

Figure 7: Comparison of the fit of the model forces to the experimental reaction force in circumferential and axial direction ( $f_{11}$ and $f_{22}$ respectively) and the last iteration (constrained mixture, CM fit). The grey dots represent the experimental data obtained from a planar biaxial test on ovine pulmonary artery tissue, the dashed red line is the classical model data and the full blue line is the constrained mixture model data. The forces are given in function of the measured circumferential and axial stretches during the test $\left(\lambda_{11}\right.$ and $\left.\lambda_{22}\right)$ for three loading ratios between both directions.

\subsubsection{Comparison of the classical and constrained mixture modeling approach}

Figure 8 gives an overview of the Cauchy stresses across the thickness of the arterial wall of the considered sheep pulmonary artery. These stresses are obtained from two different modeling approaches, as explained in section 2.5 .2 the constrained mixture approach and the classical, stress-free reference approach. 
In both cases, the stresses are given at diastolic state as well as at systolic state.

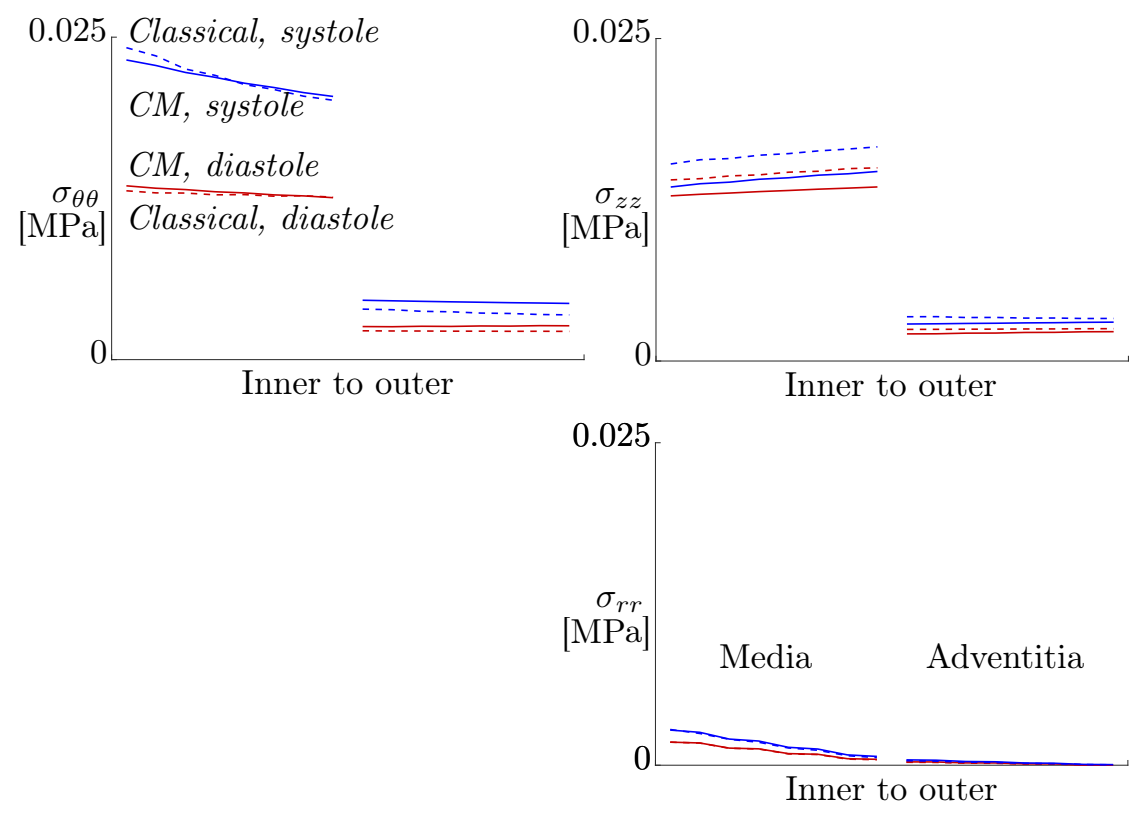

Figure 8: Comparison of the constrained mixture and classical modeling approach. The full lines represent the Cauchy stresses across the thickness of the wall for the constrained mixture approach. The dashed lines represent the same stresses obtained with the classical modeling approach. Stresses are shown at diastole and systole, i.e. an inner pressure of $2 \mathrm{kPa}$ and $3 \mathrm{kPa}$ respectively. $\sigma_{\theta \theta}, \sigma_{z z}$ and $\sigma_{r r}$ represent the circumferential, axial and radial Cauchy stresses.

\section{Discussion}

The objective of this study was to investigate how material parameters for

360 arterial tissue are affected by the introduction of the constrained mixture modeling approach. An efficient method able to provide these parameters based on planar biaxial test data was introduced, addressing the need for reliable material parameters to be used in e.g. growth and remodeling algorithms based on the constrained mixture theory. This method was verified and applied to ac- 
stresses and axial prestress in the arterial wall is compared to the constrained mixture modeling approach.

\subsection{Verification study}

Table 3 demonstrates the general performance of the CMC fitting approach

\subsection{Application to experimental data}

After verifying the iterative CMC parameter fitting approach with numerically constructed test data, the approach was also tested with actual planar biaxial test data. A diastolic geometry was build in Abaqus, where the in vivo 

iterations might not outweigh the higher computational cost, such that the convergence criterion may be made less strict, depending on the accuracy sought for.

\subsection{Comparison of the classical and constrained mixture modeling approach}

Figure 7 shows the model and experimental data. An offset from the zero force at stretch 1 can be observed. This is caused by a preload applied during the biaxial test to avoid sagging of the sample at the start, leading to a deviation to the model at low stresses. However, this artefact does not affect the aim of the present study to compare the classical parameter fitting approach with the new study, is necessary to correct for it in an accurate and straightforward manner.

The model data obtained from the classical approach matches the experimental data slightly better than at the final fit, although the difference is minimal. It was observed that the final fit was sensitive to the value for $g_{e, a x}$ and that a 420 good choice is crucial to obtain a material model that matches the experiments. It was set to a realistic value of axial prestretch. However, no exact value for the specific sample was available. Also $g_{c}$ affects the results. It was estimated at 1.1, corresponding to an approximate value found in mouse carotid arteries [28, which is possibly inaccurate for sheep pulmonary arteries. Performing 
experimental tests on the stretch state of collagen would be beneficial for the reliability.

Figure 8 shows the resulting stress states. Note that the sudden drop of stress between media and adventitia arises from the difference in stiffness. It can be observed that both models show very similar wall stresses. The circumferential

430 Cauchy stress in the medial layer is almost constant through the thickness. The homogenization of the wall stress is even clearer in the results of the constrained mixture model, showing its strength in representing residual stresses. Three possible reasons for the small difference between both models are listed. Firstly, this is partly due to a different fit of both models to the experimental data, such that the stress-stretch response is not the same. A second reason is the assumption of a stress-free radially cut state in the classical approach. From the results of the FE model following the constrained mixture approach, it can be seen that the stresses in the radially cut state are not exactly zero but approximately two orders of magnitude smaller than at diastole. Thirdly, the deformations from the diastolic state to the radially cut state, i.e. $\boldsymbol{F}_{\text {release }}$ and $\boldsymbol{F}_{\text {open }}$ are approximated and averaged over the element layers when integrated in the constrained mixture parameter fitting. On the other hand, no approximation was needed going from the stress-free state to the diastolic state in the classical FE model. A similarity between both modeling approaches is the fact that the ${ }_{445}$ radially cut state is considered to match the planar state at the beginning of the tensile test. In neither of the cases, a flattening deformation is considered.

Both modeling approaches yield similar results. Neither approach can be easily validated due to the apparent lack of a ground truth material state. However, the constrained mixture approach offers the advantage that no stress-free reference geometry is required. Therefore, it is preferred over the classical modeling method.

\subsection{Implications of results}

Bellini et al. [28] developed an improved mechanical model for arterial tissue based on histological and clinical data. It enables to capture the effects of 
the residual stresses while considering a known in vivo reference state. Several studies have suggested, both experimentally and computationally, that this constrained mixture modeling approach reliably predicts residual stresses and the mechanical behavior of arterial tissue [28, 36, 40, 44. Moreover, it provides an elegant framework for the growth and remodeling algorithms in soft tissues that are microstructurally relevant. However, to our best knowledge, no such material parameters from planar biaxial tests have been obtained before.

The aim of the present study was to provide an alternative planar biaxial tensile parameter fitting method, hypothesizing that the constrained mixture approach requires an alternative set of material parameters because of a shift in 465 energy contribution of the different constituents due to their different deformation states. The results of the verification study show a clear difference between classical and CMC parameter sets, while figure 7 shows a similar fit of the experimental data. These findings are in agreement with those of Mousavi and Avril [40. However, they found an increase in elastin stiffness and decrease in 470 collagen stiffness for the constrained mixture model. This cannot be generally concluded from the present results, presumably due to the following differences in research approach. First, they use a slightly different constitutive model, with four fiber families, where no fiber distribution is considered. Second, the present study uses biaxial tensile test data, as opposed to uniaxial test data in 475 multiple directions, possibly yielding a different constitutive behavior. In any case, given the clear difference, but unclear relation between the classical and CMC material parameter sets, the use of this developed iterative parameter fitting approach is of great importance to define a reliable constitutive model for arterial tissue based on the constrained mixture theory.

\subsection{Limitations and future work}

A number of limitations can be attributed to the presently defined parameter fitting approach. First, the constrained mixture model considers an in vivo reference state, such that all deformations with respect to that state need to be considered. However, one of these, i.e. between the radially cut configuration 

from FE simulations is not straightforward. We assume this to be reasonable due to small flattening stretches and negligible stresses that are often counteracted by gravity in practice. A second limitation is related to $g_{c}$ and $g_{e, a x}$. These deposition stretches are assumed to be fixed and known. Unreported tests show reliable deposition stretch values are deemed important. However, experimental methods to determine them are scarce. To our knowledge, only Bellini et al. 28. have reported a value for $g_{c}$. They refer to Ferruzzi et al. 45, who were able to experimentally distinguish the stress-free state of collagen from the unloaded 495 arterial configuration. Bellini et al. fine-tuned these approximate values by optimizing the fit of model data to experimental data. The parameter $g_{a x}$ is closely related to the overall axial prestretch that can be measured upon excision of an artery. If necessary, $g_{c}$ and $g_{e, a x}$ can be considered as extra parameters to be fitted. In that case, overparameterization may be avoided by obtaining the fiber angles and dispersion from histological data, such that no more than five parameters need to be fitted.

The method was verified by the numerical construction of mechanical test data, obtained from the FE simulation of an ideal planar biaxial test. It must be noted that this approach is only valid if the chosen constitutive behavior is a good description of the behavior of arterial tissue. The transverse behavior of the GOH model has been found to be unrealistic [46, 47]. Moreover, the implemented tension-compression switch has been criticised earlier [4], such that improved material models must be considered. Also the choice of parameter dependence between the medial and adventitial layer might be unrealistic. 510 Nevertheless, we hypothesize that the proposed parameter fitting method is independent of the chosen material model. The parameter dependencies were chosen based on approximate elastic versus fiber content in both layers and help to reduce the number of parameters to be fitted.

The constrained mixture model is microstructurally motivated [28] and a 
[40], such that it is assumed to be valid in the present study. However, an idealized planar biaxial test yields a uniform stress state per material layer, unattainable during an actual test. Inhomogeneities arise from nonideal boundary conditions. This impedes the obtention of the correct material parameters as compared to the ideal situation. Fehervary et al. [49] proposed an alternative parameter fitting approach to handle these inhomogeneities. Future work should be directed at integrating this approach with the currently proposed approach.

The current approach assumes an ideal cylindrical geometry and a resulting homogeneous stress state per element layer. No regional diameter, thickness, or material variations are taken into account. Future work will be directed towards nonideal geometries, where a patch of material will be virtually cut out of a patient-specific geometry.

\section{Conclusion}

The constrained mixture theory for the modeling of the arterial wall considers constituent-specific deformations and an in vivo reference state. We assume that this approach requires different material parameters. A framework for an iterative method to obtain these parameters based on planar biaxial test data was introduced, verified and tested. This study showed that the iterative fitting approach reliably converges towards the desired CMC material parameters. It can also be seen that, for specific test data sets, these parameters must be distinguished from classically obtained material parameters. Indeed, both fitting approaches yield different material parameters, such that FE models following the classical or the constrained mixture modeling theory require an adequate set of material parameters. In a first application of the iterative fitting method to actual experimental data, clear convergence occurs towards a set of CMC material parameters. These parameters, used in a FE model based on the constrained mixture approach are an important step towards patient-specific modeling of arteries, as well as reliable growth and remodeling algorithms. 
Acknowledgements.

545

This work was supported by a doctoral grant strategic basic research (SB $1 \mathrm{~S} 35316 \mathrm{~N})$ to JV, a doctoral fellowship $(11 \mathrm{~A} 6519 \mathrm{~N})$ to LM and a postdoctoral fellowship (PDO/012) to NF of the Research Foundation-Flanders (FWO). Support was also provided by KU Leuven through a category 2 research project (C2-ADAPT). SA is grateful to ERC for the ERC-2014-CoG BIOLOCHANICS grant.

\section{Appendix A: Implementation of the algorithm to find elastin deposi- tion stretches}

The iterative finite element method, described in section 2.2 of the manuscript, is constructed as follows. In a first step, an arterial geometry is pressurized. In all following steps, the applied pressure is kept constant, while the stretch state of the material is updated until the geometry has returned to its original shape. At each simulation step, a UMAT subroutine is called in which the elastin deposition stretch tensor is updated. This tensor $\boldsymbol{G}_{e}$ is stored in state dependent variables (STATEV). This subroutine provides the current deformation gradient and requires a resulting Cauchy stress (STRESS) and a Jacobian Matrix (DDSDDE).

At the end of each step, a URDFIL subroutine is called. Within URDFIL, the utility routine POSFIL is called to read from the results file with DBFILE.

${ }_{565}$ This allows to store the present deformation gradient in a global variable $\boldsymbol{F}_{d e p}$ and to stop the simulation when convergence has been reached.

call user subroutine UMAT: if $\mathrm{STEP}==1$

endif 
$\boldsymbol{G}_{e}=$ STATEV

575 if STEP $>1$

$$
\begin{aligned}
& \boldsymbol{G}_{e}=\boldsymbol{F}_{d e p} \boldsymbol{G}_{e} \\
& \text { STATEV }=\boldsymbol{G}_{e}
\end{aligned}
$$

endif

s80 $\boldsymbol{G}_{c}=\boldsymbol{G}_{c}\left(g_{c}, \boldsymbol{M}\right)$ (see equation 6 )

$\sigma_{i j}=\frac{1}{J} \frac{\partial \Psi\left(\boldsymbol{F}, \boldsymbol{G}_{e}, \boldsymbol{G}_{c}\right)}{\partial F_{i a}} F_{j a}$ (see equation 13

$C_{i j k l}=\sigma_{i j} \delta_{k l}+\frac{1}{2}\left(\frac{\partial \sigma_{i j}}{\partial F_{k a}} F_{l a}+\frac{\partial \sigma_{i j}}{\partial F_{l a}} F_{k a}\right)$

(see Nolan et al. [50], where $\boldsymbol{C}$ represents the Consistent Tangent Matrix and $\delta_{k l}$ is the Kronecker delta)

585

STRESS $=\boldsymbol{\sigma}($ convert $3 \times 3$ tensor to $6 \times 1)$

DDSDDE $=\boldsymbol{C}($ convert $3 \times 3 \times 3 \times 3$ tensor to $6 \times 6)$

call user subroutine URDFIL:

read $\boldsymbol{F}$ from results file (deformation gradient)

$\boldsymbol{F}_{\text {dep }}=\boldsymbol{F}$

595

read $U_{11}, U_{22}, U_{33}$ from results file (nodal displacement)

$U_{a v}=\sum_{n} \sqrt{U_{11, n}+U_{22, n}+U_{33, n}}$ (average nodal displacement over all nodes $n$ )

if $U_{a v}<$ Tol (Tol set to $1 \%$ of the wall thickness)

600

STOP simulation

endif 


\section{References}

[1] P. Dobrin, T. Canfield, S. Sinha, Development of longitudinal retraction of carotid arteries in neonatal dogs, Experientia 31 (11) (1975) 1295-1296.

[2] D. H. Bergel, Viscoelastic properties of the arterial wall, Ph.D. thesis (1960).

[3] R. N. Vaishnav, J. Vossoughi, Estimation of residual strains in aortic segments, in: Biomedical Engineering II: Recent Developments, 1983, pp. 330333. doi:10.1016/B978-0-08-030145-7.50078-7.

[4] C. J. Chuong, Y. C. Fung, Three-dimensional stress distribution in arteries., J Biomech Eng-T ASME 105 (3) (1983) 268-274. doi:10.1115/1.3138417

[5] C. J. Chuong, Y. C. Fung, On residual stresses in arteries., Journal of biomechanical engineering 108 (2) (1986) 189-92. URL http: //www .ncbi.nlm.nih.gov/pubmed/3079517

[6] A. Rachev, S. Greenwald, Residual strains in conduit arteries, Journal of Biomechanics 36 (5) (2003) 661-670. doi:10.1016/S0021-9290(02) $00444-\mathrm{X}$

[7] S. Q. Liu, Y. C. Fung, Relationship between hypertension, hypertrophy, and opening angle of zero-stress state of arteries following aortic constriction., Journal of biomechanical engineering 111 (4) (1989) 325-335. doi:10.1115/1.3168386.

[8] R. N. Vaishnav, J. Vossoughi, Residual stress and strain in aortic segments, Journal of Biomechanics 20 (3). doi:10.1016/0021-9290(87)90290-9.

${ }_{625}$ [9] M. K. Hong, J. Vossoughi, C. C. Haudenschild, S. C. Wong, B. D. Zuckerman, M. B. Leon, Vascular effects of diet-induced hypercalcemia after balloon artery injury in giant Flemish rabbits, American Heart Journal 130 (4) (1995) 758-764. doi:10.1016/0002-8703(95)90074-8 
[10] T. Matsumoto, K. Hayashi, K. Ide, Residual strain and local strain distributions in the rabbit atherosclerotic aorta, Journal of Biomechanics 28 (10) (1995) 1207-1217. doi:10.1016/0021-9290(94)00179-8.

[11] J. Vossoughi, H. Hedjazi, F. Borris, Intimal residual stress and strain in large arteries, in: ASME Bioengineering Conference, New York, 1993, pp. $434-437$.

[12] L. A. Taber, D. W. Eggers, Theoretical study of stress-modulated growth in the aorta, Journal of Theoretical Biology 180 (4) (1996) 343-357. doi: 10.1006/jtbi.1996.0107.

[13] G. A. Holzapfel, T. C. Gasser, R. W. Ogden, A new constitutive framework for arterial wall mechanics and a comparative study of material models,

640 Journal of Elasticity 61 (1-3) (2000) 1-48. arXiv:A:1010835316564, doi: $10.1023 / \mathrm{A}: 1010835316564$

[14] L. A. Taber, J. D. Humphrey, Stress-Modulated Growth, Residual Stress, and Vascular Heterogeneity, Journal of Biomechanical Engineering 123 (6) (2001) 528. doi:10.1115/1.1412451.

645 URL http://biomechanical.asmedigitalcollection.asme.org/ article. aspx?articleid=1476131

[15] T. C. Gasser, C. J. Schulze-Bauer, G. Holzapfel, A Three-dimensional Finite Element Model for Arterial Clamping, Journal of Biomechanical Engineering 124 (4) (2002) 355. doi:10.1115/1.1485284. URL http://link . aip.org/link/JBENDY/v124/i4/p355/s1\{\&\}Agg=doi

[16] M. L. Raghavan, S. Trivedi, A. Nagaraj, D. D. McPherson, K. B. Chandran, Three-dimensional finite element analysis of residual stress in arteries, (1) Annals of Biomedical Engineering 32 (2) (2004) 257-263. doi:10.1023/B: ABME.0000012745.05794.32

[17] D. Balzani, J. Schröder, D. Gross, Numerical simulation of residual stresses 
in arterial walls, Computational Materials Science 39 (1 SPEC. ISS.) (2007)

117-123. doi:10.1016/j.commatsci.2005.11.014.

[18] N. Famaey, G. Sommer, J. Vander Sloten, G. A. Holzapfel, Arterial clamping: finite element simulation and in vivo validation., Journal of the Mechanical Behavior of Biomedical Materials 12 (2012) 107-118. doi: 10.1016/j.jmbbm.2012.03.010.

URL http://dx.doi .org/10.1016/j.jmbbm.2012.03.010

[19] S. de Putter, B. J. Wolters, M. C. Rutten, M. Breeuwer, F. A. Gerritsen, F. N. van de Vosse, Patient-specific initial wall stress in abdominal aortic

665

[20] H. Weisbecker, D. M. Pierce, G. A. Holzapfel, A generalized prestressing algorithm for finite element simulations of preloaded geometries with application to the aorta, International Journal for Numerical Methods in Biomedical Engineering 30 (9) (2014) 857-872. arXiv:NIHMS150003, doi:10.1002/cnm.2632.

[21] J. Bols, J. Degroote, B. Trachet, B. Verhegghe, P. Segers, J. Vierendeels, A computational method to assess the in vivo stresses and unloaded configuration of patient-specific blood vessels, Journal of Computational and Applied

[22] M. L. Raghavan, B. Ma, M. F. Fillinger, Non-invasive determination of zero-pressure geometry of arterial aneurysms, Annals of Biomedical Engineering 34 (9) (2006) 1414-1419. doi:10.1007/s10439-006-9115-7.

[23] M. W. Gee, C. Reeps, H. H. Eckstein, W. A. Wall, Prestressing in finite deformation abdominal aortic aneurysm simulation, Journal of Biomechanics 42 (11) (2009) 1732-1739. doi:10.1016/j.jbiomech.2009.04.016.

[24] M. W. Gee, C. Förster, W. A. Wall, A computational strategy for prestressing patient-specific biomechanical problems under finite deformation, 
International Journal for Numerical Methods in Biomedical Engineering 26 (1) (2010) 52-72. arXiv:NIHMS150003, doi:10.1002/cnm.1236

[25] Y. C. Fung, What are the residual stresses doing in our blood vessels?, Annals of Biomedical Engineering 19 (3) (1991) 237-249. doi:10.1007/ BF02584301.

[26] P. J. Zeller, T. C. Skalak, Contribution of individual structural components in determining the zero-stress state in small arteries, Journal of Vascular Research 35 (1) (1998) 8-17. doi:10.1159/000025560.

[27] E. C. Davis, Elastic lamina growth in the developing mouse aorta., The journal of histochemistry and cytochemistry : official journal of the Histochemistry Society 43 (11) (1995) 1115-23. doi:10.1177/43.11.7560894. URL http://www.ncbi.nlm.nih.gov/pubmed/7560894

[28] C. Bellini, J. Ferruzzi, S. Roccabianca, E. S. Di Martino, J. D. Humphrey, a A Microstructurally Motivated Model of Arterial Wall Mechanics with Mechanobiological Implications, Annals of Biomedical Engineering 42 (3) (2014) 488-502. doi:10.1007/s10439-013-0928-x. URL http: //link . springer.com/10.1007/s10439-013-0928-x

[29] J. D. Humphrey, K. R. Rajagopal, A Constrained Mixture Model For Growth And Remodelling Of Soft Tissues, Mathematical Models and Methods in Applied Sciences 12 (03) (2002) 407-430. arXiv:0036013702, doi:10.1142/S0218202502001714. S0218202502001714

[30] N. Famaey, J. Vastmans, H. Fehervary, L. Maes, E. Vanderveken, F. Rega, S. J. Mousavi, S. Avril, Numerical simulation of arterial remodeling in pulmonary autografts, ZAMM - Journal of Applied Mathematics and Me710 chanics / Zeitschrift für Angewandte Mathematik und Mechanik 98 (12) (2018) 2239-2257. doi:10.1002/zamm.201700351.

URL http://doi.wiley.com/10.1002/zamm.201700351 
[31] F. A. Braeu, A. Seitz, R. C. Aydin, C. J. Cyron, Homogenized constrained mixture models for anisotropic volumetric growth and remodeling, Biomechanics and Modeling in Mechanobiologydoi:10.1007/ s10237-016-0859-1.

[32] C. J. Cyron, R. C. Aydin, J. D. Humphrey, A homogenized constrained mixture (and mechanical analog) model for growth and remodeling of soft tissue, Biomechanics and Modeling in Mechanobiology (2016) 1-15doi: $10.1007 / \mathrm{s} 10237-016-0770-9$

[33] P. N. Watton, N. A. Hill, Evolving mechanical properties of a model of abdominal aortic aneurysm, Biomechanics and Modeling in Mechanobiologydoi:10.1007/s10237-007-0115-9.

[34] S. Zeinali-Davarani, S. Baek, Medical image-based simulation of abdom725 inal aortic aneurysm growth, Mechanics Research Communicationsdoi: 10.1016/j.mechrescom.2012.01.008.

[35] S. Baek, K. R. Rajagopal, J. D. Humphrey, A Theoretical Model of Enlarging Intracranial Fusiform Aneurysms, Journal of Biomechanical EngineeringarXiv:33645723580, doi:10.1115/1.2132374.

[36] A. Valentín, J. D. Humphrey, G. A. Holzapfel, A finite element-based constrained mixture implementation for arterial growth, remodeling, and adaptation: Theory and numerical verification, International Journal for Numerical Methods in Biomedical Engineering 29 (8) (2013) 822-849. arXiv:NIHMS150003, doi:10.1002/cnm. 2555

${ }_{335}$ [37] C. Alberto Figueroa, S. Baek, C. A. Taylor, J. D. Humphrey, A computational framework for fluid-solid-growth modeling in cardiovascular sim-

1 ulations, Computer Methods in Applied Mechanics and Engineeringdoi: $10.1016 /$ j.cma.2008.09.013

[38] T. C. Gasser, R. W. Ogden, G. A. Holzapfel, Hyperelastic modelling of arterial layers with distributed collagen fibre orientations, Journal of The 
Royal Society Interface 3 (6) (2006) 15-35. doi:10.1098/rsif.2005.0073.

(1)

[40] S. J. Mousavi, S. Avril, Patient-specific stress analyses in the ascending thoracic aorta using a finite-element implementation of the constrained mixture theory, Biomechanics and Modeling in Mechanobiology 16 (5) (2017) 1765-1777. doi:10.1007/s10237-017-0918-2.

[41] J. Vastmans, H. Fehervary, P. Verbrugghe, T. Verbelen, E. Vanderveken, J. Vander Sloten, T. Treasure, F. Rega, N. Famaey, Biomechanical evaluation of a personalized external aortic root support applied in the Ross procedure, Journal of the Mechanical Behavior of Biomedical Materials 78 (2018) 164-174. doi:10.1016/j.jmbbm.2017.11.018.

[42] E. Vanderveken, J. Vastmans, T. Verbelen, P. Verbrugghe, N. Famaey, E. Verbeken, T. Treasure, F. Rega, Reinforcing the pulmonary artery autograft in the aortic position with a textile mesh: a histological evaluation, Interactive CardioVascular and Thoracic Surgery 27 (4) (2018) 566-573. doi:10.1093/icvts/ivy134 URL http://www.ncbi.nlm.nih.gov/pubmed/29912400https: //academic.oup.com/icvts/article/27/4/566/4975496

[43] V. Pratt, Direct least-squares fitting of algebraic surfaces, ACM SIGGRAPH Computer Graphics 21 (4) (1987) 145-152. doi:10.1145/37402. 37420

URL http://portal . acm.org/citation. cfm?doid=37402.37420 
[44] L. Cardamone, A. Valentín, J. F. Eberth, J. D. Humphrey, Origin of axial prestretch and residual stress in arteries, Biomechanics and Modeling in Mechanobiology 8 (6) (2009) 431-446. arXiv:NIHMS150003, doi:10.1007/ s10237-008-0146-x.

[45] J. Ferruzzi, M. J. Collins, A. T. Yeh, J. D. Humphrey, Mechanical assessment of elastin integrity in fibrillin-1-deficient carotid arteries: Implications for Marfan syndrome, Cardiovascular Researchdoi:10.1093/cvr/cvr195

[46] P. Skacel, J. Bursa, Poisson's ratio of arterial wall - Inconsistency of constitutive models with experimental data, Journal of the Mechanical Behavior of Biomedical Materialsdoi:10.1016/j.jmbbm.2015.09.029.

[47] M. Latorre, X. Romero, F. J. Montáns, The relevance of transverse deformation effects in modeling soft biological tissues, International Journal of Solids and Structuresdoi:10.1016/j.ijsolstr.2016.08.006.

[48] G. A. Holzapfel, R. W. Ogden, On the tension-compression switch in soft fibrous solids, European Journal of Mechanics, A/Solidsdoi:10.1016/j. euromechsol.2014.09.005.

[49] H. Fehervary, M. Smoljkić, J. Vander Sloten, N. Famaey, Planar biaxial 口 testing of soft biological tissue using rakes: A critical analysis of protocol and fitting process, Journal of the Mechanical Behavior of Biomedical Materials 61 (2016) 135-151. doi:10.1016/j.jmbbm.2016.01.011. URL www.elsevier.com/locate/jmbbmwww.sciencedirect.comhttp:// dx.doi.org/10.1016/j.jmbbm.2016.01.011

[50] D. R. Nolan, A. L. Gower, M. Destrade, R. W. Ogden, J. P. McGarry, A robust anisotropic hyperelastic formulation for the modelling of soft tissue, Journal of the Mechanical Behavior of Biomedical Materials 39 (2014) 4860. doi:10.1016/j.jmbbm.2014.06.016. 